\title{
On the dynamical origin of the ICM metallicity evolution
}

\author{
Sofia A. Cora, ${ }^{1,2 \star}$ Luca Tornatore, ${ }^{3,6}$ Paolo Tozzi ${ }^{4,5,6}$ and Klaus Dolag ${ }^{7}$ \\ ${ }^{1}$ Facultad de Ciencias Astronómicas y Geofísicas de la Universidad Nacional de La Plata and Instituto de Astrofísica de La Plata, \\ Observatorio Astronómico, Paseo del Bosque S/N, 1900 La Plata, Argentina \\ ${ }^{2}$ Consejo Nacional de Investigaciones Científicas y Técnicas, Rivadavia 1917, Buenos Aires, Argentina \\ ${ }^{3}$ SISSA - International School for Advanced Studies, via Beirut 4, I-43100 Trieste, Italy \\ ${ }^{4}$ Dipartamento di Astronomia dell'Universita di Trieste, via G.B. Tiepolo 11, I-34131, Trieste, Italy \\ ${ }^{5}$ INFN - Istituto Nazionale di Fisica Nucleare, Trieste, Italy \\ ${ }^{6}$ INAF - Istituto Nazionale di Astrofisica, Trieste, Italy \\ ${ }^{7}$ Max-Planck-Institut für Astrophysik, Karl-Schwarzschild-Strasse 1, 85740 Garching bei München, Germany
}

Accepted 2008 February 2. Received 2008 January 7; in original form 2007 June 26

\begin{abstract}
We present a study on the origin of the metallicity evolution of the intra-cluster medium (ICM) by applying a semi-analytic model of galaxy formation to $N$-body/smoothed particle hydrodynamic (SPH) non-radiative numerical simulations of clusters of galaxies. The semi-analytic model includes gas cooling, star formation, supernovae feedback and metal enrichment, and is linked to the diffuse gas of the underlying simulations so that the chemical properties of gas particles are dynamically and consistently generated from stars in the galaxies. This hybrid model lets us have information on the spatial distribution of metals in the ICM. The results obtained for a set of clusters with virial masses of $\sim 1.5 \times 10^{15} h^{-1} \mathrm{M}_{\odot}$ contribute to the theoretical interpretation of recent observational X-ray data, which indicate a decrease of the average iron content of the intra-cluster gas with increasing redshift. We find that this evolution arises mainly as a result of a progressive increase of the iron abundance within $\sim 0.15 R_{\mathrm{vir}}$. The clusters have been considerably enriched by $z \sim 1$ with very low contribution from recent star formation. Low entropy gas that has been enriched at high redshift sinks to the cluster centre contributing to the evolution of the metallicity profiles.
\end{abstract}

Key words: methods: numerical - galaxies: clusters: general - cosmology: observations $\mathrm{X}$-rays: galaxies: clusters.

\section{INTRODUCTION}

The intra-cluster medium (ICM) is a hot $\left(\sim 10^{7} \mathrm{~K}\right)$ and diffuse gas contained within the deep potential well of clusters of galaxies, which constitute the largest virialized structures of the Universe. This gas radiates energy through thermal bremsstrahlung, which is detected in the X-ray band of the electromagnetic spectrum, characterized by the presence of emission lines from highly ionized iron (Fe XXV and Fe XXVI) at 6.6-7 keV. These lines allow to obtain information about the metallicity of the ICM; the average Fe abundance is measured to be around $0.5 \mathrm{Z}_{\odot}$ in the central regions of local $\mathrm{X}$-ray clusters. ${ }^{1}$

Supernovae explosions are the typical sources of metals that contaminate the ICM, being generated through subsequent episodes

\footnotetext{
^E-mail: sacora@fcaglp.unlp.edu.ar

${ }^{1}$ We note that this value is different from the one found in the literature, which is based on the photosferic solar Fe abundance published in Anders \& Grevesse (1989). We are using the more recent solar value of iron abundance by number $(\mathrm{Fe} / \mathrm{H}) \odot=2.82 \times 10^{-5}$ by Asplund et al. (2005).
}

of star formation in cluster galaxies (e.g. Renzini 1997), although other sources may have a non-negligible contribution to the metal budget of the ICM, like hypernovae associated with population type III stars (Loewenstein 2001) and intra-cluster stars (Zaritsky, Gonzalez \& Zabludoff 2004). The metals produced enrich the ICM by different processes that affect the heavy element distribution, like galactic winds (Heckman et al. 2000; Springel \& Hernquist 2003), ram pressure stripping (Kapferer et al. 2007) and active galactic nuclei (Moll et al. 2006). The knowledge of the history of the chemical enrichment of the ICM contributes to our understanding on the way and epoch in which the galaxy clusters have formed, the history of star formation in cluster galaxies and the associated supernovae rates, and the physical processes involved in the diffusion of energy and metals within the hot intra-cluster gas. Observations of radial abundance profiles of different elements (De Grandi \& Molendi 2001; Tamura et al. 2004; Vikhlinin et al. 2005) provide valuable constraints on the physical processes involved in the chemical enrichment of the ICM, especially those related to the feedback mechanisms that inject metals into the diffuse phase. 
A recent analysis of Chandra X-ray spectra of 56 clusters within the redshift range $0.3 \lesssim z \lesssim 1.3$ spanning temperatures $3 \lesssim k T \lesssim$ $18 \mathrm{keV}$ allows us to trace the evolution of the iron content of the ICM (Balestra et al. 2007). The results are based on the estimated average emission-weighted (EW) iron abundance of the ICM within the inner region of the cluster, delimited by $\sim 15-30$ per cent of the virial radius, $R_{\mathrm{vir}}$. They find that, for $z \gtrsim 0.5$, the mean abundance of the ICM is approximately constant, with a value of $Z_{\mathrm{Fe}} \approx 0.4 \mathrm{Z}_{\odot}$. However, at lower redshifts $(0.3 \lesssim z \lesssim 0.5)$, the $\mathrm{EW}$ iron abundance is significantly higher, reaching a value of $Z_{\mathrm{Fe}} \approx 0.64 \mathrm{Z}_{\odot}$. The parametrization of these results implies that the iron abundance at the present epoch is a factor of $\sim 2$ larger than at $z \simeq 1$. These results are supported by the more recent study made by Maughan et al. (2008) based on a larger sample of galaxy clusters at $0.1 \lesssim z \lesssim$ 1.3 observed with Chandra ACIS-I.

From the current observed ICM metallicity evolution, it is not clear whether it arises as a result of a change in the iron content of the intra-cluster gas due to enhanced star formation, or as a consequence of redistribution of metals within the central regions; the latter would be favoured by dynamical processes which transfer the chemically enriched gas from the intergalactic medium of the cluster galaxies to the hot intra-cluster gas.

Since measurements of ICM abundances are EW, the mean abundance of the intra-cluster gas contained within a given radius is biased to higher values when considering the central regions of the clusters. This bias is expected to be higher in cool-core clusters because of the peaks in their surface brightness and iron abundance that characterize them (e.g. De Grandi \& Molendi 2001). However, the observed evolution of the ICM iron abundance cannot be entirely attributed to a possible decrease of the fraction of cool-core clusters at high redshift (Balestra et al. 2007; Maughan et al. 2008).

Some studies on the origin of the central peak in the metal distribution of clusters with a cool core suggest that it has been likely produced by the brightest cluster galaxy after the cluster was assembled (Böhringer et al. 2004; De Grandi et al. 2004). For this mechanism to be effective, large enrichment times (4-10 Gyr) are necessary. This conclusion has been achieved from the combination of observational results and population synthesis and chemical enrichment models. This possible mechanism for the formation of the central peak has been complemented with the effect of the AGNinduced flows as possible transportation processes that have mixed the metals into the ICM (Rebusco et al. 2005; Roediger et al. 2007), trying to explain the observed difference between the broad ICM metallicity profiles and the much narrower stellar light profile of the central galaxy.

Different theoretical approaches have been used so far aimed at studying the processes of metal enrichment of the ICM. By using observed and modelled rates of Type Ia supernovae (SNe Ia) and core-collapsed supernovae (SNe CC), Ettori (2005) has evaluated their contribution to the history of metal accumulation in the ICM, finding that they provide a good qualitative agreement with observations of the overall decline in abundance with redshift, thanks to the large delay times for SNe Ia adopted. However, the total amount of iron is systematically lower than observed values in the redshift range $0 \lesssim z \lesssim 1$. This model indicates that half of the iron mass observed locally is produced by $\mathrm{SNe}$ Ia, with $\mathrm{SNe} \mathrm{CC}$ products becoming dominant at higher redshifts. Different conclusions are obtained by Loewenstein (2006) who uses a similar kind of model, implying instead that synthesis of cluster iron was dominated by SNe CC and/or SN Ia with short delay times whose progenitors originated during a phase of rapid, top-heavy star formation.
The impact on the ICM chemical evolution of environmental effects, such as ram pressure, and tidal or viscous stripping, has also been analysed. Calura, Matteucci \& Tozzi (2007) use chemical evolution models applied to the different morphological types of cluster galaxies. They find that iron-enriched gas ejected by galactic winds arising from ellipticals accounts for the ICM iron abundances at $z \gtrsim 0.5$, whereas the gas stripped from the disc galaxies turning into S0 through interaction of the interstellar medium with the ICM accounts for the ICM metallicity at $z \lesssim 0.5$. The joint contribution of these processes can explain the increase in the ICM abundance in the low-redshift range observed by Balestra et al. (2007). By using a completely different approach based on a combination of $N$-body with a phenomenological galaxy formation model, which provides the ejected galactic matter that is then included into a hydrodynamic ICM simulation, Kapferer et al. (2007) find that the mass loss triggered by ram pressure stripping is more dominant than galactic winds since higher redshifts $(z \lesssim 2)$; however, their enrichment model cannot account for the observed evolution of the mean ICM metallicity with redshift.

From a hybrid model that combines an $\mathrm{N}$-body/smoothed particle hydrodynamic (SPH) non-radiative simulation of a galaxy cluster with a semi-analytic model of galaxy formation, Cora (2006) has shown that dynamical effects play a major role in the development of central iron abundance peaks. In the proposed scenario, the intergalactic medium of infalling cluster galaxies, that has been primarily enriched at high redshifts and then transferred to the ICM, is subsequently driven to the cluster centre by bulk motions in the intra-cluster gas.

Using cosmological hydrodynamical simulations, Tornatore et al. (2007) also find that gas dynamical effects, related to gas mixing, galactic winds and ram pressure stripping, play an important role in determining the distribution and relative abundance of different chemical species, being the typical age of the ICM enrichment of $z \simeq 0.5$. However, the influence of an excess of star formation at low redshift is more important than the one obtained with the hybrid model of Cora (2006), which gives quite low recent star formation for cluster galaxies.

For the theoretical interpretation of the observational results concerning the ICM metallicity evolution, we apply here the hybrid model of the chemical enrichment of the ICM implemented by Cora (2006). Although this hybrid approach does not couple metal production to the gas actually cooling in radiative simulations (e.g. Tornatore et al. 2007), its novel feature is that the chemical properties of the diffuse gas in the underlying non-radiative $\mathrm{N}$-body/SPH cluster simulations are generated from metals ejected from the galaxies, consistent with the modelling of the semi-analytic model. These metals are then carried around and mixed by the hydrodynamic processes during cluster formation, allowing us to study the evolution of the spatial distribution of metals in the ICM. We compare the results of our hybrid model with the mean iron abundances of the ICM recently determined by Balestra et al. (2007), with the aim of investigating the connection between the development of the chemical abundance patterns that characterize the ICM and the observed average metallicity evolution.

This paper is organized as follows. Section 2 briefly describes the hybrid model used to study the ICM chemical enrichment, presenting the changes applied to the version described in Cora (2006), mainly related to the distribution of metals among gas particles; it also summarizes the properties of the hydrodynamical simulations used. Section 3 contains the analysis of the evolution of the ICM chemical enrichment considering both radial abundance profiles (Section 3.1) and mean metallicities (Section 3.2) of the main 
cluster progenitor at different redshifts. In Section 4, we summarize our conclusions.

\section{MODEL OF THE ICM CHEMICAL ENRICHMENT}

We consider a hybrid model for studying the chemical enrichment of the ICM, which consists of a combination of non-radiative cosmological $N$-body/SPH simulations that contain galaxy clusters and a semi-analytic model of galaxy formation. The important feature of this hybrid model is the link between semi-analytic model results and the chemical enrichment of the diffuse gas component of the underlying $\mathrm{N}$-body/SPH simulation. We pollute gas particles with metals ejected from the galaxies that have been generated by the semi-analytic model. By enriching the gas particles locally around galaxies, we can account for the spreading and mixing of metals by hydrodynamical processes, thereby obtaining a model for the evolution of the spatial distribution of metals in the ICM.

\subsection{Semi-analytic model of galaxy formation}

The semi-analytic model used here is based on previous works (Springel et al. 2001; De Lucia, Kauffmann \& White 2004), but was extended with a new chemical implementation that tracks the abundance of different species produced by different sources. We briefly describe here the modelling of the circulation of metals among the different baryonic components. Details of this model are given in Cora (2006). The simplicity of the semi-analytic model has the advantage of reaching a larger dynamic range than fully self-consistent hydro-simulations, at a far smaller computational cost. In particular, it allows us to explore more easily the range of parameters that characterize appropriate chemical enrichment models. The long cooling time of the bulk of the gas in rich clusters justifies the assumption of a non-radiative gas in the $N$-body/SPH simulations. However, this time-scale becomes smaller than the Hubble time in the core of the cluster, where the densities are higher. Here is where the semi-analytic model plays an important part, taking into account radiative cooling, star formation, and chemical enrichment and energetic feedback from galaxies by including the effect of supernovae explosions.

Dark matter haloes and substructures that emerge in the simulation are tracked by the semi-analytic code and are used to generate the galaxy population (Springel et al. 2001). In this subhalo scheme, the virial mass of a dark matter substructure is defined simply as the sum of the mass of its particles, and the virial radius is estimated by assuming that the subhalo has an overdensity of 200 with respect to the critical density. Since each particle in the $N$-body/SPH simulations is split into dark matter and gas, the identification of dark matter haloes is based on the dark matter particles with their masses corrected for the cosmic baryon fraction. This is done to get haloes defined as close as possible to pure dark matter runs, on which semi-analytic models are usually calibrated, but still allows to make use of the hydrodynamic treatment for the distribution of metals ejected by galaxies.

We assume that the hot gas always has a distribution that parallels that of the dark matter halo. Its mass is initially given by the baryon fraction of the virial mass of the dark matter halo, and is subsequently modified by gas cooling, star formation and feedback processes. Stars can contaminate the cold and hot gas because of mass loss during their stellar evolution and metal ejection at the end of their lives. The hot gas has primordial abundances initially (76 per cent of hydrogen and 24 per cent of helium), but becomes chemically enriched as a result of the direct ejecta from stellar mass loss, and the transfer of contaminated cold gas to the hot phase that is reheated by supernovae explosions. This chemical enrichment has a strong influence on the amount of hot gas that can cool, since we are using metal-dependent cooling rates (Sutherland \& Dopita 1993). This process in turn influences the star formation activity which is ultimately responsible for the chemical pollution.

The chemical model implemented in our semi-analytic code considers mass losses from stars in different mass ranges. Massive stars give raise to core-collapse supernovae, which include those of Type Ib/c and II, the latter being the most abundant ones. We adopt stellar yields as given from models of Marigo (2001) for low- and intermediate-mass stars $\left(0.8 \mathrm{M}_{\odot} \lesssim M \lesssim 5-8 \mathrm{M}_{\odot}\right)$ and Portinari, Chiosi \& Bressan (1998) for quasi-massive $\left(5 \mathrm{M}_{\odot} \lesssim\right.$ $\left.M \lesssim 8 \mathrm{M}_{\odot}\right)$ and massive stars $\left(8 \mathrm{M}_{\odot} \lesssim M \lesssim 120 \mathrm{M}_{\odot}\right)$. Ejecta from Type Ia supernovae are also included, considering the nucleosynthesis prescriptions from the updated model W7 by Iwamoto et al. (1999). We use the stellar lifetime given by Padovani \& Matteucci (1993) to model the return time-scale of the ejecta from all sources considered, being specially relevant for the single stars in the low- and intermediate-mass range and for the progenitors of $\mathrm{SNe}$ Ia, that are characterized by a long delay time from the formation of the progenitor to the supernova explosion. We adopt the single degenerate model to estimate the SNe Ia rate, following the scheme of Greggio \& Renzini (1983), where Type Ia supernovae originate in binary systems whose components have masses between 0.8 and $8 \mathrm{M} \odot$. Calculations are based on the formalism described in Lia, Portinari \& Carraro (2002), but assuming that core-collapse supernovae originate from single stars with masses larger than $8 \mathrm{M}_{\odot}$.

The mass range of secondary stars in binary systems gives explosion times for SNe Ia comprising between $\sim 2.9 \times 10^{7}$ and $\sim 1.4 \times 10^{10} \mathrm{yr}$, with the SNe Ia rate reaching a maximum within $\sim 0.1-0.7$ Gyr for a single stellar population. Thus, the ejection rate of elements produced by SNe Ia reaches a maximum at $z \sim 3-5$, while the contribution of SNe CC to the ICM chemical enrichment peaks at higher redshifts $(z \sim 5-7)$ since SNe CC rate closely follows the star formation rate as a result of the short lifetimes of the stars involved. The peaks in the mass ejection rates are followed by a strong decline at lower redshifts for both types of $\mathrm{SNe}$, such that the ongoing chemical contamination is quite low at $z=0$ (Cora 2006). The relative contribution of $\mathrm{SNe} \mathrm{CC}$ and $\mathrm{SNe}$ Ia to the iron content of the cluster is already one-third at $z \sim 2$, as given by the accumulated iron masses provided by both sources.

Some aspects of the semi-analytic model have been modified with respect to the version described in Cora (2006). We consider here a Salpeter initial mass function (IMF) normalized between 0.1 and $100 \mathrm{M}_{\odot}$. In order to obtain a mass-metallicity relation whose evolution with redshift approaches to the observed one (Erb et al. 2006), half of the mass ejected by galaxies because of mass loss through stellar winds or supernovae explosions is transferred directly to the hot phase instead of being first incorporated to the cold gas. In this way, the cold gas associated to each galaxy is more gradually contaminated, instead of already achieving at high redshifts the higher metallicitiy values that correspond to the present epoch.

The free parameters of the model regulate the way in which gas cooling, star formation, supernovae feedback and galaxy mergers proceed, and determine the circulation of metals among the different baryonic components. They have been carefully tuned in Cora (2006) to satisfy numerous observational constraints (Milky Way properties, the luminosity function, the Tully-Fisher, colour-magnitude and mass-metallicity relations). The feedback 
efficiency parameter that regulates the amount of cold gas that is reheated by core-collapse supernovae is now assigned a smaller value of 0.1 that allows to recover a better agreement of model results with the observed slope of the Tully-Fisher relation.

\subsection{Non-radiative $N$-body/SPH cluster simulations}

The semi-analytic model is applied to cosmological hydrodynamical simulations. We consider a set of three simulated galaxy clusters, having virial mass in the range $\simeq(1-2) \times 10^{15} h^{-1} \mathrm{M}_{\odot}$. These clusters have been initially selected from a dark matter simulation of a cosmological box, having size of $479 h^{-1} \mathrm{Mpc}$ (Yoshida, Sheth \& Diaferio 2001), for a cosmological model $\Omega_{\mathrm{m}}=0.3, \Omega_{\Lambda}=0.7$, $H_{0}=70 \mathrm{~km} \mathrm{~s}^{-1} \mathrm{Mpc}^{-1}, \Omega_{\mathrm{b}}=0.039$ for the baryon density parameter and $\sigma_{8}=0.9$ for the normalization of the power spectrum. The Lagrangian regions surrounding the three selected clusters have been re-simulated at higher mass resolution by applying the Zoomed Initial Condition technique (Tormen, Bouchet \& White 1997). The mass resolution is the same for all simulations, with masses of dark matter and gas particle of $m_{\mathrm{dm}}=1.13 \times 10^{9} h^{-1} \mathrm{M}_{\odot}$ and $m_{\text {gas }}=1.69 \times 10^{8} h^{-1} \mathrm{M}_{\odot}$, respectively. As for the force resolution, the Plummer-equivalent gravitational softening is fixed at $\varepsilon=5 h^{-1} \mathrm{Kpc}$ in physical units at redshift $z \leqslant 5$, while it switches to comoving units at higher redshifts. Table 1 gives the number of high-resolution dark matter particles of each simulation, which is equal to the number of gas particles, and the virial properties of its most massive cluster. The simulations have been carried out using the TREE-SPH GADGET-2 code (Springel 2005). GADGET-2 is a parallel TREE + SPH code with fully adaptive time-stepping, which includes an integration scheme which explicitly conserves energy and entropy (Springel \& Hernquist 2002). The simulations considered here include only non-radiative physics and the original formulation of artificial viscosity within SPH. General properties of these simulated clusters, such as radial profiles of velocity dispersion of dark matter and gas particles, and of gas temperature, density and entropy, are given in Dolag et al. (2005).

\subsection{Hybrid model of the ICM metal enrichment}

The key physical processes that lead to the evolution of the iron content of the ICM are the production of metals and their deposition in the gas. The former is controlled by the prescriptions included in the semi-analytic model, while the latter depends on the way in which chemical elements generated by galaxies in the semi-analytic model are distributed among gas particles in the $N$-body/SPH cluster simulation, which thus carry the information on the spatial distribution of chemical and thermodynamical properties of the ICM. The metal

Table 1. Number of high-resolution dark matter particles, $N_{\mathrm{hr}}$, of the nonradiative cosmological hydro-simulations, and the virial mass, $M_{\mathrm{vir}}$, temperature, $T_{\mathrm{vir}}$ and radius, $R_{\mathrm{vir}}$, of the most massive cluster in each simulation. The simulation name used in Dolag et al. (2005) is given between parentheses.

\begin{tabular}{lcccc}
\hline Cluster & $N_{\mathrm{hr}}$ & $\begin{array}{c}M_{\text {vir }} \\
\left(h^{-1} \mathrm{M}_{\odot}\right)\end{array}$ & $\begin{array}{c}k T_{\text {vir }} \\
(\mathrm{keV})\end{array}$ & $\begin{array}{c}R_{\text {vir }} \\
\left(h^{-1} \mathrm{Mpc}\right)\end{array}$ \\
\hline C1 (g51) & 2219034 & $1.3 \times 10^{15}$ & 7.8 & 2.3 \\
C2 (g1) & 4937886 & $1.5 \times 10^{15}$ & 8.4 & 2.4 \\
C3 (g8) & 5602561 & $1.7 \times 10^{15}$ & 8.1 & 2.8 \\
\hline
\end{tabular}

deposition results from a complex combination of different processes that include powerful outflows from star-forming galaxies, generated by supernovae explosions and winds from young massive stars, dynamical stripping and gas mixing. We model the deposition of metals in a simple way. For each snapshot of the simulation, we identify a set of $N_{\text {gas }}$ gas particles within a sphere characterized by an enrichment radius, $R_{\text {enrich }}$, centred on each galaxy. Metals ejected by each galaxy are spread among the $N_{\text {gas }}$ gas particles. Both the choice of $R_{\text {enrich }}$ and the procedure used to distribute the chemical elements have been modified with respect to the version presented in Cora (2006). In that work, metals were uniformly distributed within a radius of $100 h^{-1} \mathrm{Kpc}$ for all galaxies.

In the present implementation, the mass of iron ejected by the galaxy is distributed among its corresponding set of $N_{\text {gas }}$ gas particles according to the same kernel used for SPH calculations in the $N$-body/SPH simulation, that has been obtained from a $B$-spline. Besides, we now consider a physically motivated model for determining $R_{\text {enrich }}$ based on a treatment of galactic winds (Bertone, Stoehr \& White 2005). It follows the expansion of supernova-driven superbubbles around several hundred thousand galaxies formed in a region of space with diameter $52 h^{-1} \mathrm{Kpc}$ and mean density close to the mean density of the universe. About half of the enclosed galaxies at $z=0$ are field galaxies, while the rest are in groups and poor clusters. They solve the equation of motion for a spherical astrophysical blastwave to follow the evolution of winds after they escape the visible regions of galaxies. Although the history of each wind depends on the properties of the parent galaxy and on the environment where the wind expands, Bertone et al. (2005) find small scatter in the distribution of the shock radii for different parameter choices of the model at a given redshift and as a function of the stellar mass of the galaxy. This ensures that mean quantities of global properties of the winds well represent the general trend of the whole population.

Considering the results of Bertone et al. (2005), we adopt the dependence of the mean bubble radius for all galaxies blowing a wind at $z=3$, as a function of the stellar mass, $M_{\star}$, of the host galaxies (see their fig. 3). This dependence varies according to the parameters chosen for the wind model, such that the bubble radius ranges from $\approx 100$ to $400 h^{-1} \mathrm{Kpc}$ for galaxies with $M_{\star} \approx 10^{11} \mathrm{M}_{\odot}$. In order to explain the origin of the observed evolution of the iron content of the ICM, we first have to reproduce the iron spatial distribution at $z=0$. We find that the curve for which galaxies with stellar mass $M_{\star} \approx 10^{11} \mathrm{M}_{\odot}$ have a bubble radius of $100 \mathrm{~h}^{-1} \mathrm{Kpc}$ allows to recover radial iron abundance profiles in good agreement with observations, as shown in Section 3.1. We fit this curve with a parametrization given by

$R_{\text {enrich }}=-623.13+133.01 \log M_{\star}-6.13 \log ^{2} M_{\star}$.

Using a larger bubble radius for massive galaxies, or the same radius for all galaxies (fixed at $100 \mathrm{~h}^{-1} \mathrm{Kpc}$ as in Cora 2006), gives place to radial iron abundance profiles flatter than observed.

It is important to note that we are not considering the evolution of the bubble radius given by the treatment of Bertone et al. (2005), but only the relationship between this radius with the stellar mass of the host galaxy at a given redshift. The subsequent spreading and mixing of metals are carried out by gas particles themselves through hydrodynamical processes. The consistent implementation in our model of the equations governing the wind evolution and their impact on the chemical pollution of the ICM will be presented in a forthcoming work. 


\section{EVOLUTION OF ICM IRON CONTENT}

\subsection{Radial abundance profiles}

Observed iron abundances at different clustercentric radii for local clusters (Tamura et al. 2004; Vikhlinin et al. 2005) constitute an important constraint to our model. We evaluate its capability to represent the chemical enrichment of the ICM by comparing the radial iron abundance profiles of the ICM constructed from simulated results at redshift $z=0$ with this kind of observational data.

Radial abundance profiles are built by dividing the volume limited by the cluster virial radius into concentric spherical shells centred on the dominant galaxy of the cluster. These shells have equal width till $0.1 R_{\text {vir }}$ and are logarithmically spaced beyond this radius. For each shell, we estimate the X-ray EW mean iron abundance relative to hydrogen, using the emission measure of each gas particle defined as $\mathrm{EM} \equiv \int n_{\mathrm{p}} n_{\mathrm{e}} \mathrm{d} V$, where $n_{\mathrm{p}}$ and $n_{\mathrm{e}}$ are the proton and electron densities, respectively, and $V$ is the volume associated to the gas particle. The iron abundance is then expressed in terms of the solar value, for which we have adopted the recent calibration of Asplund et al. (2005). These mean abundances are assigned to the mean radius of each shell.

Fig. 1 shows the EW radial iron abundance profile of the ICM at $z=0$ for the three simulated clusters (thick lines). They are compared with a sample of nearby, relaxed galaxy clusters observed with Chandra (Vikhlinin et al. 2005), with a range of average temperatures similar to that of the simulated clusters. The comparison of the model abundances with observed values is shown out to $R_{500}$, which is the radius enclosing a mean overdensity of 500 with respect to the critical density at the redshift of the cluster. The local EW profile shows a good agreement with the trend traced by observations, except in the innermost radial bins, being at odds with self-consistent hydrodynamical simulations that include gas cooling. The presence of dark matter substructures with associated high-density and chemically enriched gas makes the EW abundance profiles quite noisy at distances larger than $\sim 0.2 R_{500}$ because of the strong dependence of the X-ray emissivity on the gas density. These peaks are not present in mass-weighted (MW) abundance profiles, which show almost the same trend than EW ones but are much smoother.

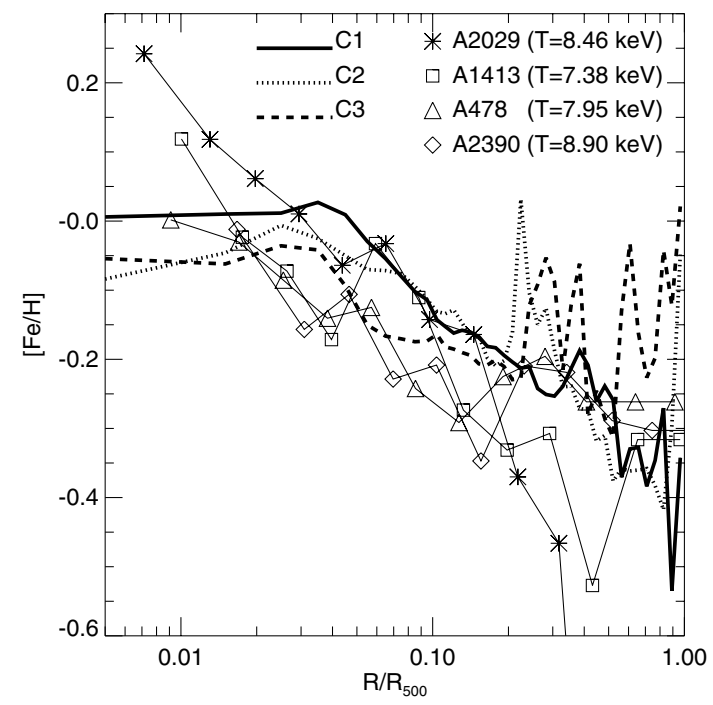

Figure 1. EW radial iron abundance profiles for the three simulated clusters (thick solid, dotted and dashed lines). Symbols represent observational data from Vikhlinin et al. (2005).

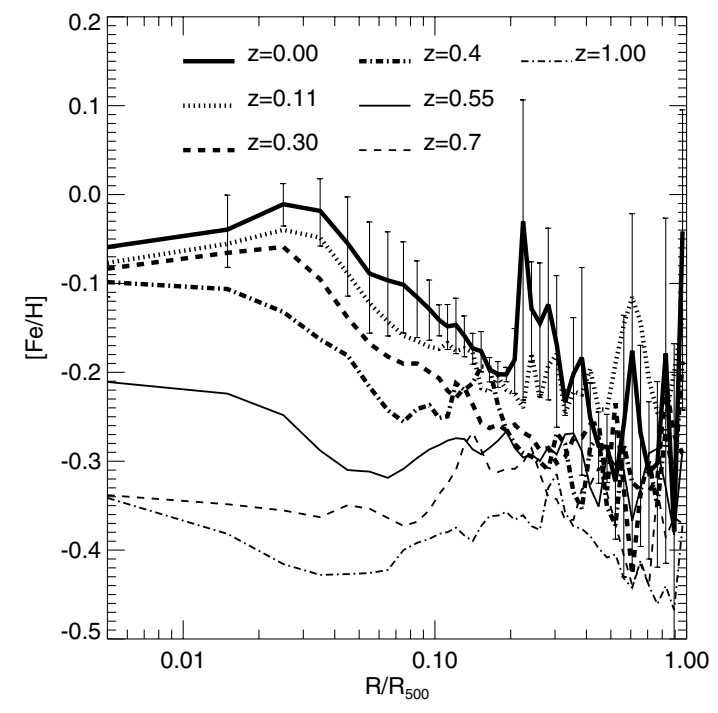

Figure 2. Evolution with redshift of EW radial iron abundance profiles. They are obtained by averaging at each concentric shell the abundance distribution of the three clusters analysed. The error bars around the average radial profile at $z=0$ denote the $1 \sigma$ standard deviation of the mean.

The evolution of the radial abundance profiles is followed by identifying the main progenitor of the cluster at different redshifts. Then, we take into account the gas particles that reside within the virial radius of these structures. The main progenitor of a given halo at each redshift is selected among the set of progenitors of that halo and is defined as the substructure with the largest number of dark matter particles. Fig. 2 shows the evolution with redshift of EW radial iron abundance profiles of the ICM. Each curve corresponds to a different redshift and has been obtained by considering the gas particles within each radial bin for all the three clusters; thus giving an average abundance distribution. The thick solid line corresponds to the present epoch, being the average of the individual radial profiles represented by the thick curves in Fig. 1.

From Fig. 2, we can appreciate that the EW iron abundances become progressively lower with increasing redshift, with the main change occurring at radii less than $\sim 0.2 R_{500}\left(\sim 0.15 R_{\mathrm{vir}}\right)$, where the profiles become flatter. At larger radii, the shape of the abundance distributions at different redshifts keeps themselves quite close to the profile at $z=0$. That is, the evolution of the radial abundance profiles from $z \sim 1$ to 0 is mainly produced by an increment of the iron content in the innermost regions of the clusters.

\subsection{Mean metallicity}

Recent results on the evolution of the iron content of the ICM based on the analysis of Chandra X-ray spectra of 56 clusters of galaxies at $z \gtrsim 0.3$ (Balestra et al. 2007) support a decrease of metallicity with redshift that can be parametrized by a power law of the form $\sim(1+z)^{-1.25}$. This observed evolution implies that the average iron content of the ICM at the present epoch is a factor of $\sim 2$ larger than at $z \simeq 1$.2. Since this analysis is restricted to the central regions $(R \simeq$ $0.15-0.3 R_{\mathrm{vir}}$ ) of clusters, the evolution of the iron content might be interpreted as a result of occurrence of evolution in the gradients of the metal distribution. This hypothesis becomes possible taking into account our results on the evolution of the EW radial iron abundance profiles shown in Fig. 2. 


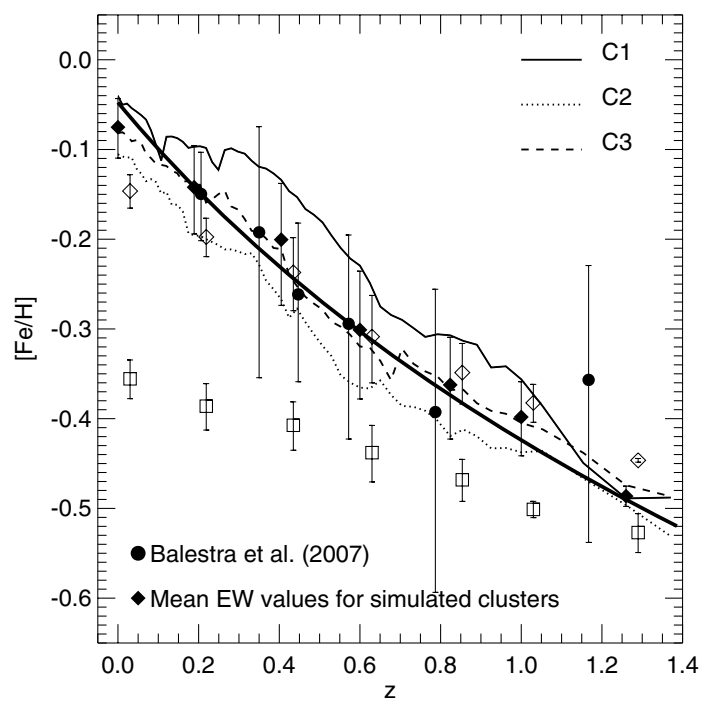

Figure 3. Evolution of the EW mean iron abundance of the main progenitors of the galaxy clusters analysed (thin lines). These mean values have been estimated using gas particles within $0.15 R_{\mathrm{vir}}$. The mean values for all the three clusters are shown at some redshifts (filled diamonds; error bars denote $1 \sigma$ confidence level). The corresponding MW mean iron abundances estimated within $0.15 R_{\mathrm{vir}}$ and $R_{\mathrm{vir}}$ are also shown (empty diamonds and empty squares, respectively). These results are compared with the observational data from Balestra et al. (2007) (filled circles) and the corresponding parametrization of the metallicity evolution with redshift (thick solid line). The observational data have been multiplied by a factor of 1.66 in order to refer the abundances to the solar values of Asplund et al. (2005).

In order to compare our results with those of Balestra et al. (2007), we estimate the mean EW iron abundance from the iron content of gas particles contained within $0.15 R_{\mathrm{vir}}$ from the centre of the cluster or of its progenitor, depending on the redshift considered. Fig. 3 shows this comparison for each of the clusters analysed, together with the corresponding mean values for all the three clusters estimated at some redshifts (filled diamonds). Observational data are represented by filled circles and the thick solid line denotes the corresponding parametrization of the metallicity evolution. Model results closely follow the trend denoted by this parametrization within the whole redshift range considered. The innermost area contained within $0.15 R_{\text {vir }}$ manifests the strongest changes in the slope of the radial abundance profiles (see Fig. 2). Thus, the decrease of the observed EW mean iron abundances with increasing redshift can be connected to the flattening of the central part of the abundance profiles.

The rate of change of the EW mean metallicity with redshift has a mild dependence with the region of the cluster considered, manifesting clear evolution even for the case where all gas within $R_{\text {vir }}$ is taken into account (not shown in the figure). The low-density and poorly contaminated gas particles lying at distances greater than $\sim 0.15 R_{\text {vir }}$ do not contribute much to the EW mean metallicity because of their low X-ray emissivity. Hence, EW mean abundances mainly provide information of the central parts of the clusters.

Instead, estimations of MW mean iron abundances give equal weight to both high and low enriched gas particles since their masses do not change in a non-radiative SPH simulation. Therefore, MW mean iron abundances strongly depend on the region of the cluster considered. Taking into account gas particles within either $0.15 R_{\text {vir }}$ or $R_{\mathrm{vir}}$ leads to MW mean metallicity evolution with smaller values when all gas contained within $R_{\text {vir }}$ is involved in the calculations.
This is a consequence of the presence of progressively less contaminated gas particles with increasing clustercentric radius. Results corresponding to $R_{\text {vir }}$ and $0.15 R_{\text {vir }}$ are shown in Fig. 3 by empty squares and empty diamonds, respectively.

As a result of the evolution of the slope of the radial abundance profiles within $0.15 R_{\mathrm{vir}}$, the MW mean metallicity does show stronger evolution with redshift only when these inner parts of the cluster are considered, becoming quite close to the behaviour given by EW values, as shown in Fig. 3. Hence, the observed metallicity evolution can be explained as being produced by the combination of two effects. On one hand, the evolution on MW mean iron abundances estimated from gas contained within $R_{\mathrm{vir}}$ indicates that there is an overall increase of the iron content within the virial radius of the main progenitor of the cluster. This is also evident from the lower values displayed by the whole radial abundance profiles as redshift increases (see Fig. 2). On top of this, we find that the evolution of the slope of the central part of the iron abundance profiles $\left(\lesssim 0.15 R_{\text {vir }}\right)$ is the key ingredient that contributes to achieve the observed evolutionary trend for $z \lesssim 1.4$.

\subsection{Understanding the evolution of the ICM metallicity}

The joint evaluation of observational and simulated results supports the fact that the observed evolution of the ICM iron content till redshift $z \simeq 1.2$ is mainly due to the evolution of the metallicity gradients of the intra-cluster gas characterized by an increase of central abundances with decreasing redshift. Both the star formation rate and the mass accretion history of the cluster may give raise to such evolution. The study carried out by Cora (2006), based on the analysis of a single simulated cluster, favours the scenario in which dynamical effects play the major role in the increment of the central abundances of the ICM, since the amount of star formation at $z \lesssim$ 0.5 is not enough to account for the observed evolution of the iron content.

Fig. 4 shows the history of metal ejection since $z \lesssim 1.4$. For the simulated cluster $\mathrm{C} 1$, we estimate the accumulated iron mass ejected into the ICM below a given redshift by galaxies contained within $0.15 R_{\mathrm{vir}}$ and $R_{\mathrm{vir}}$; it is expressed in terms of the total mass

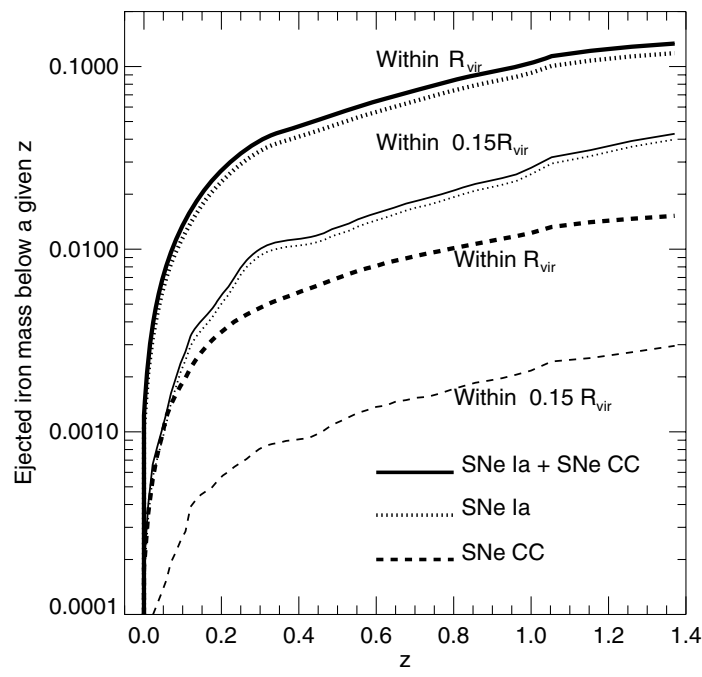

Figure 4. Evolution with redshift of iron mass ejected by galaxies within

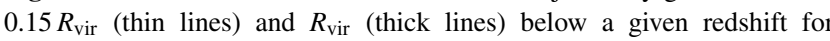
cluster $\mathrm{C} 1$, expressed in terms of the iron mass contained within those radii at $z=0$. Different types of lines indicate the joint or separate contribution of supernovae Type Ia and CC. 
of iron contained in the ICM within those radii at $z=0$. We see that, for $z \lesssim 1.4$, the accumulated iron mass ejected from galaxies within $R_{\text {vir }}$ is $\sim 10$ per cent, while this fraction is reduced to $\sim 4-5$ per cent when only galaxies within $0.15 R_{\text {vir }}$ are taken into account. In this last case, the ejected iron mass drops quite abruptly at $z \simeq 0.3$, making negligible contribution to the iron budget of the ICM within the innermost regions. We also separately show the contribution of different types of supernovae. We find that $\sim 90$ per cent of the iron mass ejected since $z<1.4$ is provided by SNe Ia.

These values of mass of iron ejected can be considered as lower limits because of the low star formation rate of cluster galaxies at low redshifts in our model. This behaviour arises as a result of the suppression of gas cooling in the central galaxies of haloes with $V_{\text {vir }}>350 \mathrm{~km} \mathrm{~s}^{-1}$ and in satellite galaxies; they simply form stars till their reservoir of cold gas is exhausted. The first condition has been imposed to avoid too massive cluster central galaxies that are not consistent with observations. The reduction or suppression of the cooling flow could be naturally produced by AGN feedback (Bower et al. 2006; Croton et al. 2006) which is not currently included in our model. As a result of the different modelling, it is not surprising to find that the iron ejected within $R_{\mathrm{vir}}$ since $z \lesssim 0.5$ is a factor of 2 smaller than the estimations obtained by the completely different approach of Calura et al. (2007), based on chemical evolution models and morphological information, or those arising from self-consistent cosmological hydrodynamical simulations (Tornatore et al. 2007), which has an excess of low-redshift star formation.

The fact that, at a low redshift, the ICM in our model receives a very small contribution of iron ejected by cluster galaxies allows us to isolate the role of dynamical processes in the evolution of the ICM abundance patterns. With the aim of understanding the way in which gas dynamics contribute to develop the iron abundance profiles, we select gas particles located within a sphere of radius $0.15 R_{\mathrm{vir}}$ centred on the cluster at $z=0$. They are tracked back to high redshifts in order to analyse the evolution of the relationship between their iron content and entropy values. Fig. 5 shows the dependence of such quantities for cluster $\mathrm{C} 1$ at redshifts $z=0,0.5$ and 1 for those gas particles that remain within the main progenitor of the cluster as the redshift increases. Dots in the entropymetallicity planes are colour-coded according to the distance of gas particles with respect to the centre of the main progenitor of the clus- ter: within $0.02 R_{\text {vir }}$ (red), in the ranges $0.02-0.05 R_{\text {vir }}$ (magenta), $0.05-0.15 R_{\text {vir }}$ (blue), $0.15-0.3 R_{\text {vir }}$ (cyan) and $0.3-1 R_{\text {vir }}$ (green). The behaviour of gas particles lying outside the virial radius of the main progenitor is presented in Fig. 6, at redshifts $z=0.1,0.5$ and 1.

The distribution in the entropy-metallicity plane of gas particles located at clustercentric distances $\lesssim 0.15 R_{\text {vir }}$ at $z=0$ (right-hand panel of Fig. 5) simply reflects the fact that the closer the particles are from the cluster centre, the lower are their entropy values, according to the entropy profile of the cluster (Dolag et al. 2005). Only $\sim 19$ per cent of these particles reside at distances $\lesssim 0.15 R_{\text {vir }}$ at $z \approx 1$ (left-hand panel of Fig. 5), while a larger percentage ( $\sim 58$ per cent) is found outside the main progenitor of the cluster (left-hand panel of Fig. 6). This behaviour is consistent with the flattening of metallicity profiles as redshift increases (see Fig. 2). Both sets of gas particles, within and outside the virial radius of the main progenitor, have already achieved a high level of enrichment by $z \approx$ 1. Around 14-15 per cent of gas associated to other progenitor haloes is characterized by low entropy values $\left(\lesssim 10 \mathrm{keV} \mathrm{cm}^{2}\right)$ which are not so common among gas particles lying within the main progenitor.

As the cluster assembles, gas particles outside the main progenitor of the cluster are incorporated into it following the infalling substructures, which are stripped in the process. At $z \approx 0.5$, the percentage of gas particles contained within the virial radius of the main progenitor increases to $\sim 63$ per cent, with $\sim 40$ lying at $\lesssim 0.15 R_{\mathrm{vir}}$. Very few particles are found outside the main progenitor at $z \approx 0.1$, as can be seen from the right-hand panel of Fig. 6 . A small fraction of gas is stripped quite early from the halo progenitors; they are then incorporated on to the cluster by some diffusive accretion mechanism, increasing their entropy as a result of the shocks suffered during the process (Dolag et al. 2005). As time passes by, the enriched low entropy gas converges to the inner regions of the cluster. The entropy profile of the ICM regulates this contribution. Although we have non-radiative simulations, the temperature profile of the ICM at $z=0$ decreases for radius smaller than 0.05 Rvir. As discussed by Churazov et al. (2003), the presence of this dense and cool core only allows the penetration of the low entropy gas attached to the infalling dark matter substructures that have been enriched at high redshift when the iron ejecta from galaxies were larger. The mass accreted on to the main progenitor at a given time may vary among the different clusters analysed because of their particular
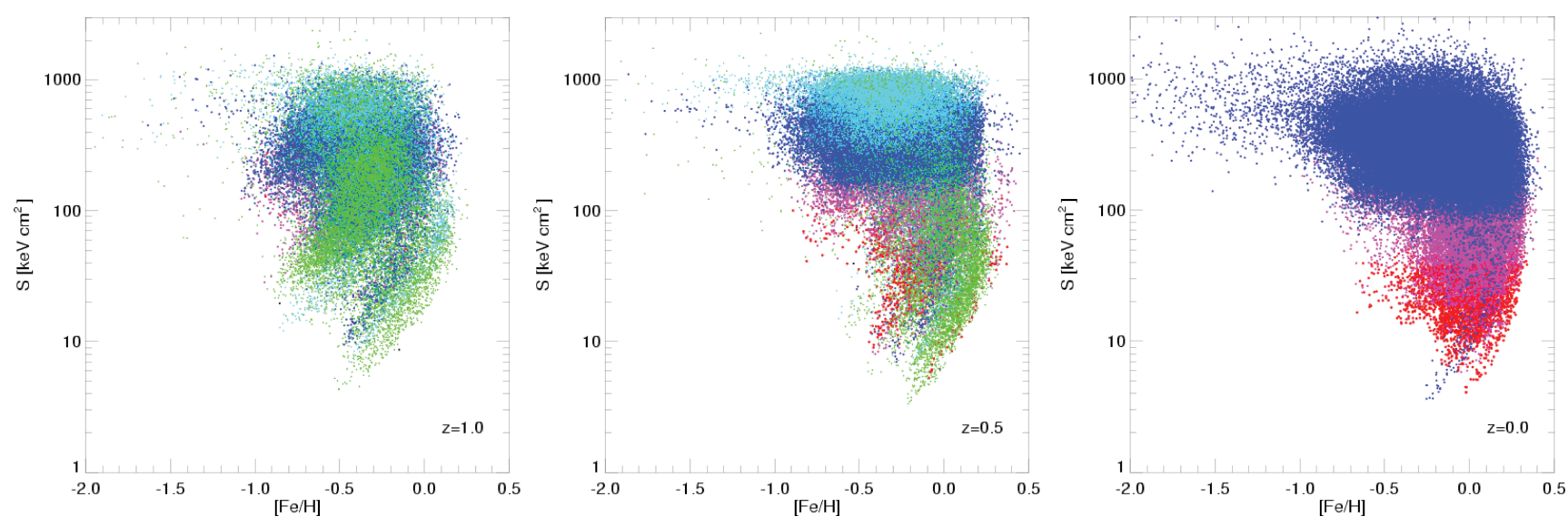

Figure 5. Evolution with redshift of the dependence between the entropy and iron abundance of gas particles that have distances from the cluster centre within a sphere of radius $0.15 R_{\mathrm{vir}}$ at $z=0$. Results correspond to cluster C1. Redshifts are indicated in each panel. Gas particles are colour-coded according to their distances with respect to the centre of the main progenitor of the cluster at each redshift: within $0.02 R_{\mathrm{vir}}$ (red), in the ranges $0.02-0.05 R_{\mathrm{vir}}$ (magenta), $0.05-0.15 R_{\text {vir }}$ (blue), $0.15-0.3 R_{\text {vir }}$ (cyan) and $0.3-1 R_{\text {vir }}$ (green). Gas particles outside $R_{\text {vir }}$ are shown in Fig. 6. 

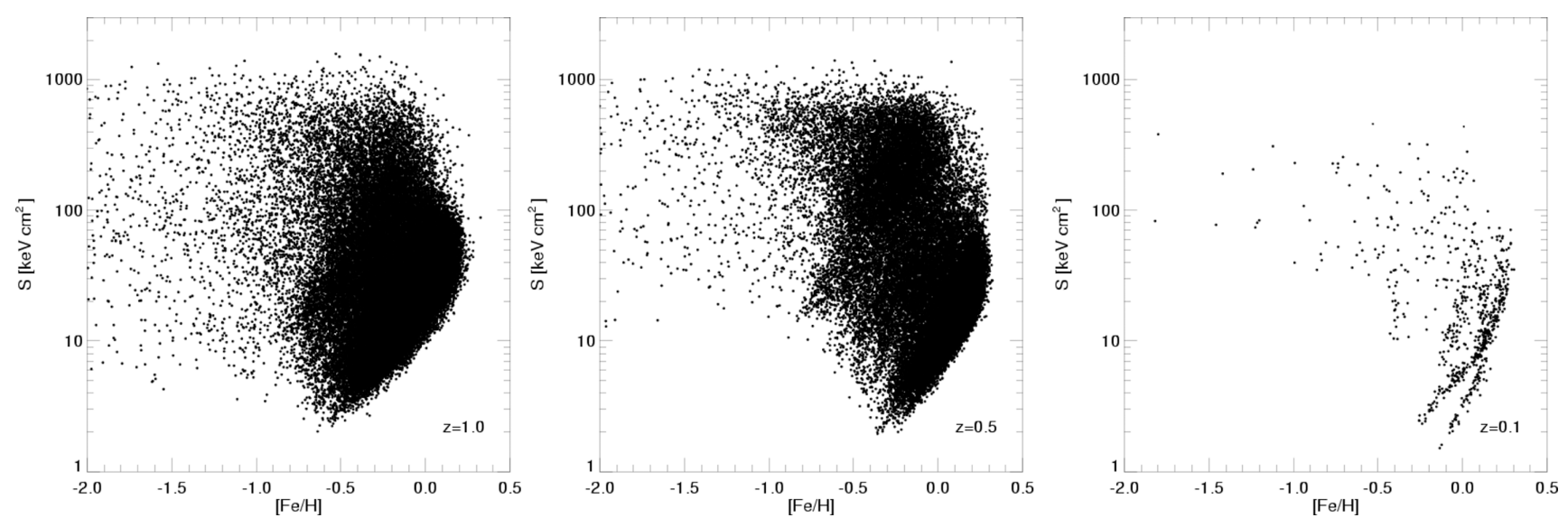

Figure 6. Evolution with redshift of the dependence between the entropy and iron abundance of gas particles that are outside the virial radius of the main progenitor of the cluster and end up within a sphere of radius $0.15 R_{\mathrm{vir}}$ centred at the cluster $\mathrm{C} 1$ at $z=0$. Redshifts are indicated in each panel.

formation history; however, the general features of the evolution in the entropy-metallicity plane is the same for all of them.

From this analysis, we find that much of the central, most highly enriched gas originates in infalling substructures that are not the main cluster progenitor. This scenario of the origin of the iron abundance pattern is supported by the results of a test case in which gas particles are contaminated only by galaxies contained within the main progenitor of the cluster. In such a restricted enrichment scheme, the iron abundances are very low and the shape of the profile becomes quite flat in comparison with the one obtained when the contribution of all galaxies is taken into account. The dynamical process that involves the sinking of enriched low entropy gas explains the evolution suffered by the metallicity profiles in their central parts, which cannot be accounted for by recent enhanced star formation activity. We can see the steepening of the profiles as a consequence of the spatial redistribution of the intra-cluster gas with different level of chemical enrichment.

\section{CONCLUSIONS}

We have applied a hybrid model for metal enrichment of the ICM that combines hydrodynamical cosmological simulations of galaxy clusters and a semi-analytic model of galaxy formation. This hybrid model has the special feature of linking the metal production of galaxies in the semi-analytic model with the chemical enrichment of gas particles in the $N$-body/SPH simulations. This allows us to follow the evolution with time of the spatial distribution of metals in the intra-cluster gas.

We have adopted a scheme for metal spreading among gas particles which is based on the dynamics of the expansion of supernovadriven superbubbles generated around galaxies (Bertone et al. 2005). We find that this physically motivated estimation of the enrichment radius satisfies observed ICM metallicity properties both locally and at high redshifts, being in good agreement with iron abundance profiles at $z=0$ and the evolution of the mean abundances with look-back time. The simulated mean metallicities within $0.15 R$ vir are in good agreement with the observations analysed by Balestra et al. (2007) in the whole redshift range considered, closely following the parametrization of observed data till $z \lesssim 1.4$. The results of this model let us conclude the following.

(i) The observed mean metallicity evolution (Balestra et al. 2007) can be explained as being produced by the combination of an overall increase of the iron content within the virial radius of the main progenitor of the cluster, and the evolution of the slope of the central part of the iron abundance profiles $\left(\lesssim 0.15 R_{\mathrm{vir}}\right)$. This result has been obtained from the analysis of both MW and EM iron abundance profiles and mean metallicities, with EW mean values mainly providing information of the central parts of the clusters.

(ii) The metallicities of gas particles that at $z=0$ end up within a region delimited by a radius of $\sim 0.15 R_{\text {vir }}$ have been already achieved between $z \sim 1$ and $\sim 0.5$, when almost half of the gas has not yet been accreted on to the main progenitor of the cluster, and the metal ejecta from galaxies was considerably higher with respect to the present epoch.

(iii) As time passes by, the enriched low entropy gas, mainly attached to infalling substructures, sinks into the cluster and is mixed there contributing to develop the iron abundance profiles. The presence of a dense and cool core allows the penetration of this low entropy gas (Churazov et al. 2003). Hence, the very central metallicity $\left(\lesssim 0.02 R_{\text {vir }}\right.$ ) originates almost exclusively from gas in infalling substructures that converge to the cluster core. This dynamical process explains the evolution suffered by the metallicity profiles in their central parts, which cannot be accounted for by recent enhanced star formation activity.

The shape of entropy profiles determines the development of the abundance profiles. The previous conclusions have been obtained for non-radiative simulations of galaxy clusters. The entropy profile changes substantially for radiative runs, as has been shown by Borgani et al. (2005), with the main change occurring within $\sim 0.1 R_{\text {vir }}$. For simulations that include gas cooling, star formation and feedback regulated by the velocity of galactic winds, the entropy profile monotonically decreases to the cluster centre, while in non-radiative simulations an almost isentropic core develops. The change in the central entropy values would only affect the limit on the entropy of the gas in infalling substructures that is allowed to penetrate the core, without affecting the general development of the profiles that we have described by the analysis of our results.

Gas cooling, star formation and feedback processes from supernovae explosions associated to galaxy formation are taken into account in the semi-analytic model. The lack of AGN feedback in our model obliges us to use simple rules for the quenching of star formation to avoid overcooling in the semi-analytical prescription, and therefore we do not have a robust control of the cooled gas fraction. The impact of AGN feedback on galaxy properties is currently being investigated (Lagos, Cora \& Padilla, in preparation); this improved semi-analytic model will be used to study the influence of 
AGN feedback on the ICM metal enrichment. In the case of coolcore clusters, the formation and evolution of the central brightest galaxy play an important role (Böhringer et al. 2004; De Grandi et al. 2004). Its effect will be properly modelled with the inclusion of AGN feedback that mainly affects star formation in massive haloes; this will allow to evaluate its influence on the development of the ICM metallicity, and to quantify the relative importance with respect to gas dynamical effects.

The semi-analytic model does not consider tidal disruption of cluster galaxies which give raise to intra-cluster stars, as shown by high-resolution $N$-body/SPH simulations (Murante et al. 2004; Sommer-Larsen, Romeo \& Portinari 2005). In our model, the potential intra-cluster stars remain within the galaxies; their ejected supernovae products are affected by the feedback process considered instead of being injected directly into the intra-cluster gas, the latter being the main advantage of the unbound component in the enrichment process of the ICM (Zaritsky et al. 2004). Thus, we can consider that our model underestimates the contribution of potential intra-cluster stars to the metal budget of the ICM. On the other hand, numerical simulations show that unbound stars (at least $\sim 10$ per cent of the cluster stellar mass) accumulate within the cluster generating intra-cluster light with a shallower radial profile than that of the bound component within $\sim 0.3 R_{\text {vir }}$; besides, they are on average older than the stars in cluster galaxies, which iron ejection rates already peak at high redshift. Hence, based on these results, we do not expect that intra-cluster stars drive a significant evolution of the ICM iron abundance profile at low redshifts.

Ram pressure stripping is another process that might change the shape of the slope of the abundance pattern in the innermost part of the ICM. Its effect is present on the least bound gas particles in the hydrodynamical simulations. However, our semi-analytic model does not currently include the ram pressure stripping on the cold gas of cluster galaxies, which would affect the cold gas reservoir available for star formation and the timing of galactic mass loss. The evaluation of the impact of this mechanism will be part of future work.

\section{ACKNOWLEDGMENTS}

We warmly thank Stefano Borgani, who proposed this research project, for largely contributing to this work with useful suggestions and comments. We acknowledge the anonymous referee for helpful remarks that improved this work. SAC is very grateful for the hospitality of the Osservatorio Astronomico di Trieste, where this project was started, and the travel grant from the National Institute for Nuclear Physics, Trieste, Italy. PT acknowledges financial contribution from contract ASI-INAF I/023/05/0 and from the PD51 INFN grant. We thank Fabio Gastaldello for helpful comments. The simulations were carried out with CPU time allocated at the 'Centro Interuniversitario del Nord-Est per il Calcolo Elettronico' (CINECA, Bologna), thanks to grants of INAF and from the University of Trieste. This project was financially supported by Fundación Antorchas, PIP 5000/2005 from Consejo Nacional de Investigaciones Científicas y Técnicas, and PICT 26049 of Agencia de Promoción Científica y Técnica, Argentine.

\section{RE F E RENCES}

Anders E., Grevesse N., 1989, Geochim. Cosmochim. Acta, 53, 197

Asplund M., Grevesse N., Sauval A. J., 2005, in Barnes T. G. III, Bash F. N., eds, ASP Conf. Ser. Vol. 336, Cosmic Abundances as Records of Stellar Evolution and Nucleosynthesis, Astron. Soc. Pac., San Francisco, p. 25
Balestra I., Tozzi P., Ettori S., Rosati P., Borgani S., Mainieri V., Norman C., Viola M., 2007, A\&A, 462, 429

Bertone S., Stoehr F., White S. D. M., 2005, MNRAS, 359, 1201

Böhringer H., Matsushita K., Churazov E., Finoguenov A., Ikebe Y., 2004, A\&A, 416, L21

Borgani S., Finoguenov A., Kay S. T., Ponman T. J., Springel V., Tozzi P., Voit G. M., 2005, MNRAS, 361, 233

Bower R. G., Benson A. J., Malbon R., Helly J. C., Frenk C. S., Baugh C. M., Cole S., Lacey C. G., 2006, MNRAS, 370, 645

Calura F., Matteucci F., Tozzi P., 2007, MNRAS, 378, L11

Cora S. A., 2006, MNRAS, 368, 1540

Croton D. J. et al., 2006, MNRAS, 365, 11

Churazov E., Forman W., Jones C., Böhringer H., 2003, ApJ, 590, 225

De Grandi S., Molendi S., 2001, ApJ, 551, 153

De Grandi S., Ettori S., Longhetti M., Molendi S., 2004, A\&A, 419, 7

De Lucia G., Kauffmann G., White S. D. M., 2004, MNRAS, 349, 1101

Dolag K., Vazza F., Brunetti G., Tormen G. G., 2005, MNRAS, 364, 753

Erb D. K., Shapley A. E., Pettini M., Steidel C. C., Reddy N. A., Adelberger K. L., 2006, ApJ, 644, 813

Ettori S., 2005, MNRAS, 262, 110

Greggio L., Renzini A., 1983, A\&A, 118, 217

Heckman T. M., Lehnert M. D., Strickland D. K., Armus L., 2000, ApJS, 129,493

Iwamoto K., Brachwitz F., Nomoto K., Kishimoto N., Umeda H., Hix W. H., Thielemann F.-K., 1999, ApJS, 125, 439

Kapferer W. et al., 2007, MNRAS, 466, 813

Lia C., Portinari L., Carraro G., 2002, MNRAS, 330, 821

Loewenstein M., 2001, ApJ, 557, L573

Loewenstein M., 2006, ApJ, 648, 230

Marigo P., 2001, A\&A, 194, 217

Maughan B. J., Jones C., Forman W., Van Speybroeck L., 2008, ApJS, 174, 117

Moll R. et al., 2007, A\&A, 463, 513

Murante G. et al., 2004, ApJ, 607, L83

Nagashima M., Lacey C., Baugh C. M., Frenk C. C., 2005, MNRAS, 358, 1247

Padovani P., Matteucci F., 1993, ApJ, 416, 26

Portinari L., Chiosi C., Bressan A., 1998, A\&A, 334, 505

Rebusco P., Churazov E., Böhringer H., Forman W., 2005, MNRAS, 359, 1041

Renzini A., 1997, ApJ, 488, 35

Roediger E., Brüggen M., Rebusco P., Böhringer H., Churazov E., 2007, MNRAS, 375, 15

Romeo A. D., Sommer-Larsen J., Portinari L., Antonuccio-Delogu V., 2006, MNRAS, 371, 548

Sommer-Larsen J., Romeo A. D., Portinari L., 2005, MNRAS, 357, 478

Springel V., 2005, MNRAS, 364, 1105

Springel V., Hernquist L., 2002, MNRAS, 333, 649

Springel V., Hernquist L., 2003, MNRAS, 339, 289

Springel V., White S. D. M., Tormen G., Kauffmann G., 2001, MNRAS, 238, 726

Sutherland R. S., Dopita M. A., 1993, ApJS, 88, 253

Tamura T., Kaastra J. S., Herder J. W. A. den, Bleeker J. A. M., Peterson J. R., 2004, A\&A, 420, 135

Tormen G., Bouchet F., White S. D. M., 1997, MNRAS, 286, 865

Tornatore L., Borgani S., Dolag K., Matteucci F., 2007, MNRAS, 382, 1050

Valdarnini R., 2003, MNRAS, 339, 1117

Vikhlinin A., Markevitch M., Murray S. S., Jones C., Forman W., Van Speybroeck L., 2005, ApJ, 628, 655

Vikhlinin A., Kravtsov A., Forman W., Jones C., Markevitch M., Murray S. S., Van Speybroeck L., 2006, ApJ, 640, 691

Yoshida N., Sheth R. K., Diaferio A., 2001, MNRAS, 328, 669

Zaritsky D., Gonzalez A. H., Zabludoff A. I., 2004, ApJ, 613, L93

This paper has been typeset from a $\mathrm{T}_{\mathrm{E}} \mathrm{X} / \mathrm{LT}_{\mathrm{E}} \mathrm{X}$ file prepared by the author. 